\title{
Determination of the charge carrier compensation mechanism in Te-doped GaAs by scanning tunneling microscopy
}

\author{
J. Gebauer ${ }^{\mathrm{a})}$ and E. R. Weber \\ Department of Materials Science and Engineering, University of California at Berkeley and Materials \\ Science Division, Lawrence Berkeley National Laboratory, Berkeley, California 94720 \\ N. D. Jäger, K. Urban, and Ph. Ebert \\ Institut für Festkörperforschung, Forschungszentrum Jülich GmbH, D-52425 Jülich, Germany
}

(Received 6 December 2002; accepted 30 January 2003)

\begin{abstract}
We identified the charge carrier compensation mechanism in Te-doped GaAs with atomically resolved scanning tunneling microscopy. Three types of defects were found: tellurium donors $\left(\mathrm{Te}_{\mathrm{As}}\right)$, Ga vacancies $\left(\mathrm{V}_{\mathrm{Ga}}\right)$, and $\mathrm{Ga}$ vacancy-donor complexes $\left(\mathrm{V}_{\mathrm{Ga}}-\mathrm{Te}_{\mathrm{As}}\right)$. We show quantitatively that the compensation in Te-doped bulk GaAs is exclusively caused by vacancydonor complexes in contrast to $\mathrm{Si}$-doped GaAs. This is explained with the Fermi-level effect as the universal mechanism leading to Ga vacancy formation in $n$-doped GaAs, and a Coulomb interaction leading to the formation of the complexes. The quantification of the carrier compensation yields a $-3 e$ charge state of $\mathrm{V}_{\mathrm{Ga}}$ in bulk GaAs. (C) 2003 American Institute of Physics.

[DOI: $10.1063 / 1.1563835]$
\end{abstract}

Semiconductor device technology depends crucially on controlled and reproducible doping. Unfortunately, the highest achievable carrier concentration is frequently limited by the formation of compensating defects during crystal growth or processing of devices. ${ }^{1}$ A technologically important example for compensation is $n$-doped GaAs, in which the carrier concentration is usually limited to the mid $-10^{18}-\mathrm{cm}^{-3}$ range. $^{2}$ A physical understanding of the compensation mechanism(s) ultimately requires a microscopic identification of all defects and dopant atoms present in the semiconductor. Scanning tunneling microscopy (STM) allows such a direct microscopic identification of individual defects and dopant atoms. ${ }^{3}$ This was shown for Si-doped GaAs, in which the $\mathrm{Si}$ atoms on $\mathrm{Ga}$ lattice sites $\left(\mathrm{Si}_{\mathrm{Ga}}\right)$ acting as donors are compensated by the consecutive formation of amphoterically incorporated $\mathrm{Si}_{\mathrm{As}}$ acceptors, neutral $\mathrm{Si}$ clusters, and $\mathrm{Ga}$ vacancy-donor complexes. ${ }^{4}$

GaAs doped with the nonamphoteric $n$-type dopant Te shows significant compensation as well, ${ }^{2}$ raising the question of the compensation mechanism. A number of different compensating defects, such as Ga vacancy-donor complexes, ${ }^{2}$ $\mathrm{Ga}_{\mathrm{As}}$ antisites, ${ }^{5}$ As interstitials, ${ }^{6}$ or amphoteric native defect complexes, ${ }^{7}$ have been suggested to be responsible for the compensation, but at present it is not clear which defect is dominating.

In this letter, we identify the compensating defects in Te-doped GaAs by atomically resolved, cross-sectional STM, which allows individual distinction, proof of presence, and quantification of the individual defects and dopant atoms. ${ }^{4,8}$ By directly observing the individual $\mathrm{Te}_{\mathrm{As}}$ donors, $\mathrm{Ga}$ vacancies $\left(\mathrm{V}_{\mathrm{Ga}}\right)$, and $\mathrm{Ga}$ vacancy-donor complexes $\left(\mathrm{V}_{\mathrm{Ga}}-\mathrm{Te}_{\mathrm{As}}\right)$ in STM images we extract their surface and bulk concentrations. A comparison with the $\mathrm{Te}$ and the carrier concentrations allows us to conclude that the compensation

${ }^{a}$ Electronic mail: JGebauer@lbl.gov is exclusively caused by $\mathrm{V}_{\mathrm{Ga}}-\mathrm{Te}_{\mathrm{As}}$ complexes. Furthermore, we find that the Ga vacancy is $-3 e$ charged in $n$-doped GaAs.

We investigated melt-grown GaAs with a Te concentration of $c_{\mathrm{Te}}=5.9 \times 10^{18} \mathrm{~cm}^{-3}$ [determined by secondary ion mass spectroscopy (SIMS)]. The electron concentration was only $n_{\mathrm{e}}=4.2 \times 10^{18} \mathrm{~cm}^{-3}$ (determined by Hall-effect measurements at $300 \mathrm{~K}$ ), pointing to the presence of compensation. The samples were cleaved in UHV (base pressure $<10^{-8} \mathrm{~Pa}$ ) perpendicular to a [110] direction. Previous difficulties in obtaining large, atomically flat cleavage planes on highly Te-doped $\mathrm{GaAs}^{9}$ were overcome by using very thin (thickness $<60 \mu \mathrm{m}$ ) samples. The cleavage surface with the defects exposed was observed by STM in the constantcurrent mode. Frequently, the same line was scanned twice with different polarities of the tunneling voltage. This allows a separate imaging of the filled As and empty Ga dangling bond states at negative and positive sample voltages, respectively. ${ }^{10}$

Figure 1 shows pairs of double polarity images of the occupied [frames (a1)-(c1)] and empty [frames (a2)-(c2)] states of the three types of defects observed on the Te-doped GaAs cleavage surfaces. No other defects except a small number of adsorbates were found. The defects can be identified as follows.

(i) $T e_{A s}$ donors: Frames (a1) and (a2) in Fig. 1 show three individual defects which exhibit a long-range elevation for both polarities of the sample voltage. This is the signature of a positive electric charge as observed for $\mathrm{Si}$ donors on GaAs. ${ }^{11,12}$ No dangling bond is missing, indicating that the defects contain no vacancies. ${ }^{3}$ The different defects labeled D1, D2, and D3, exhibit, from D1 to D3, decreasing contrast heights and alternating symmetry properties. All defect images have a $(1 \overline{1} 0)$ mirror plane, either located on a row (D1 and D3) or between rows (D2) of As-derived occupied states (Fig. 2, solid lines). In the empty state images, the same location of the mirror plane is observed; that is, it is located 

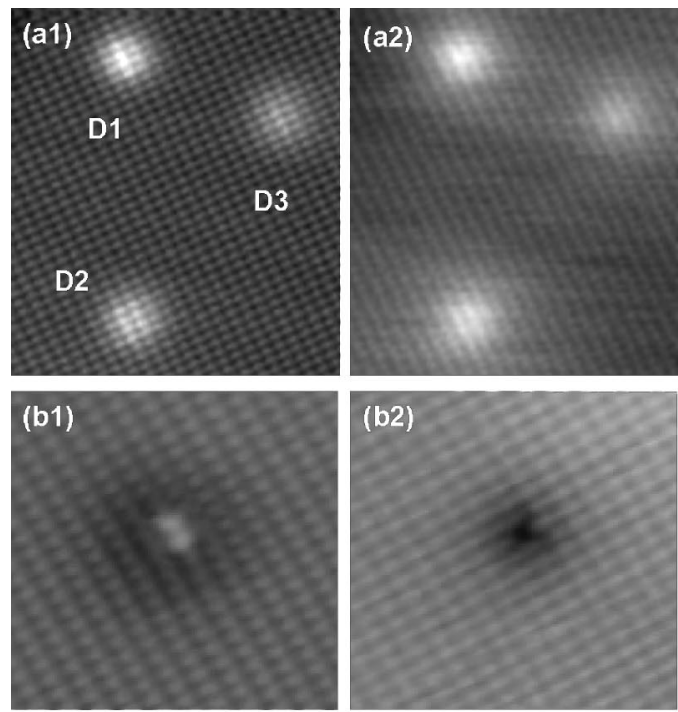

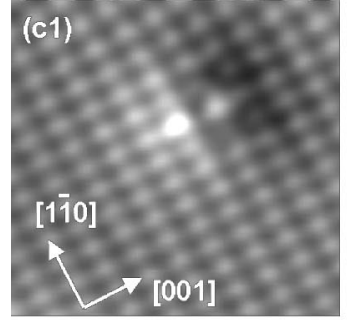

occupied states

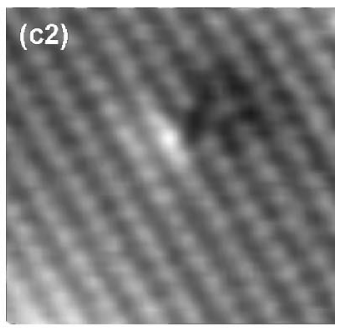

empty states
FIG. 1. Constant-current STM images of the occupied (left frames) and empty (right frames) states of the three defects found on Te-doped GaAs cleavage surfaces. Frames (a1) and (a2) show three $\mathrm{Te}_{\mathrm{As}}^{+}$donors (labeled D1-D3) in different subsurface layers (the number indicates the layer). (b1) and (b2) show a Ga vacancy, and (c1) and (c2) a Ga vacancy-donor complex. The tunneling voltages are (a1) $-1.44 \mathrm{~V},(\mathrm{a} 2)+1.44 \mathrm{~V}$, (b1) $-1.5 \mathrm{~V}$, (b2) $+1.5 \mathrm{~V}$, (c1) $-1.44 \mathrm{~V}$, and (c2) $+1.44 \mathrm{~V}$.

between rows (D1 and D3), and on top of a row (D2) of the Ga-derived states (Fig. 2, dashed lines).

The features just described point to positively charged substitutional defects in different subsurface layers. The dopant D1 with the highest contrast is located in the surface

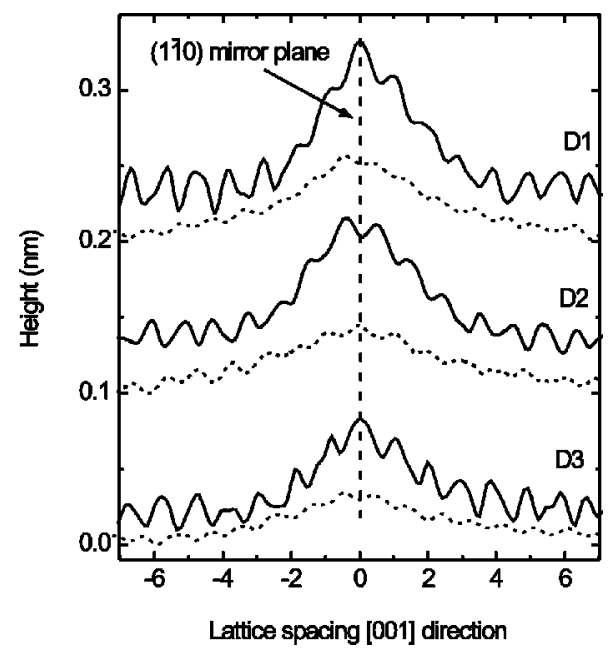

FIG. 2. Height profiles along the [001] direction through the occupied (solid lines) and empty (dashed lines) states images of the $\mathrm{Te}_{\mathrm{As}}$ donors labeled D1-D3 in Fig. 1 [frames (a1) and (a2)]. The profiles are offset along the height axis for clarity. The (1 $\overline{1} 0)$ mirror plane showing the defect symmetry is indicated.

Downloaded 21 Dec 2006 to 134.94.122.39. Redistribution subject to AIP license or copyright, see http://apl.aip.org/apl/copyright.jsp

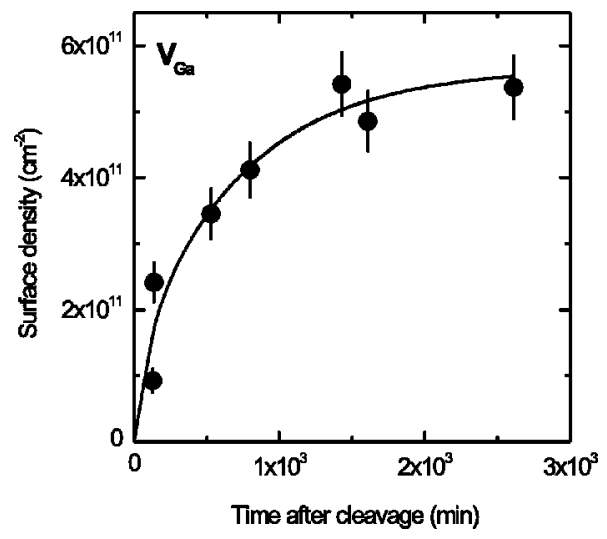

FIG. 3. Concentration of Ga vacancies on the cleavage surface of Te-doped $\mathrm{GaAs}$ as a function of time after cleavage. The density of $\mathrm{V}_{\mathrm{Ga}}$ directly after cleavage $(t=0)$ is estimated by extrapolation (solid line) to be zero, indicating that the Ga vacancies are surface related only.

layer. The alternating symmetry properties for the dopants D1-D3 are compatible with donors incorporated on As lattice sites in the first three layers. ${ }^{12}$ Thus, we attribute defects D1-D3 in Fig. 1 (a1) and (a2) to $\mathrm{Te}_{\mathrm{As}}$ donors.

The volume concentration of the donors was determined from the area concentration for each of the three layers. The concentration was independent on time, suggesting that these are indeed volume defects. The average concentration was $(6.1 \pm 0.3) \times 10^{18} \mathrm{~cm}^{-3}$ in agreement with the total Te concentration measured by SIMS, confirming the assignment. In addition, this shows that $\mathrm{Te}$ is incorporated exclusively on the As sublattice.

(ii) Ga vacancies: Images of the second defect type are shown in Fig. 1, frames (b1) and (b2). The defect exhibits a bright contrast at negative and dark contrast at positive voltage. This is the signature of a negative electric charge. ${ }^{3} \mathrm{~A}$ dangling bond is missing in the empty states image (b2), and the two neighboring dangling bonds are raised in the filled state image (b1). This defect has been shown previously to be a negatively charged, isolated Ga vacancy. 3,13

Because $\mathrm{V}_{\mathrm{Ga}}$ is an acceptor, it could potentially explain the observed compensation. However, the concentration of $\mathrm{V}_{\mathrm{Ga}}$ increases with time (Fig. 3). This effect has been attributed to a Fermi-level effect driven Langmuir desorption. ${ }^{4,14}$ The extrapolation back to the cleavage time (solid line in Fig. 3) yields a vanishing concentration, showing that the isolated $\mathrm{Ga}$ vacancies form on the surface only after cleavage. They are thus not present in the bulk and have no influence on the compensation.

(iii) $V_{G a}-T e_{A s}$ complexes: The third defect exhibits a missing empty dangling bond [Fig. 1 (c2)] indicating that it contains a Ga vacancy. The symmetry is, however, distinctly different from that of the isolated $\mathrm{V}_{\mathrm{Ga}}$ shown in Fig. 1 (b2), showing that the defect is rather a complex. In the filled state image [Fig. 1 (c1)], no dangling bond is missing, but the dangling bond neighboring the vacancy is raised. It does not calized dipole character like $\mathrm{Si}_{\mathrm{Ga}}-\mathrm{V}_{\mathrm{As}}{ }^{15}$ or $\mathrm{Zn}_{\mathrm{Ga}}-\mathrm{V}_{\mathrm{As}}{ }^{14,16}$ complexes. Thus, the defect is assigned to a neutral $\mathrm{Te}_{\mathrm{As}}$-donor-Ga-vacancy complex surrounded by a dipole screening field. The complex concentration did not change with time. Therefore, the $\mathrm{V}_{\mathrm{Ga}}-\mathrm{Te}_{\mathrm{As}}$ complexes are bulk defects exposed on the surface. Their concentration was exhibit a long-range elevation or depression but rather a lo- 
$(6 \pm 2) \times 10^{17} \mathrm{~cm}^{-3}$. Our assignment matches the interpretation of previous positron annihilation studies of Te-doped GaAs. ${ }^{17}$ The STM experiments here allow a direct microscopic identification of the defects probed by positron annihilation as $\mathrm{V}_{\mathrm{Ga}}-\mathrm{Te}_{\mathrm{As}}$ complexes.

With the identification of all defects, we can now discuss the compensation mechanism in Te-doped GaAs. The only acceptors possibly present in the bulk are the $\mathrm{V}_{\mathrm{Ga}}-\mathrm{Te}_{\mathrm{As}}$ complexes. In contrast to $\mathrm{Si}$-doped $\mathrm{GaAs},{ }^{4}$ neither substitutional acceptors such as $\mathrm{Si}_{\mathrm{As}}$ nor dopant precipitates were found. Thus, the compensation must be exclusively caused by $\mathrm{V}_{\mathrm{Ga}}-\mathrm{Te}_{\mathrm{As}}$. On this basis, we can quantitatively discuss the compensation. From the difference $\left(c_{\mathrm{Te}}-n_{\mathrm{e}}\right)$, one can deduce that the concentration of compensated donors is $1.7 \times 10^{18} \mathrm{~cm}^{-3}$. The concentration of the $\mathrm{V}_{\mathrm{Ga}}-\mathrm{Te}_{\mathrm{As}}$ complexes is, however, one third of that value. Thus, the complex cannot be neutral in the bulk, as observed on the surface by STM. The isolated Ga vacancy is expected to be either threefold ${ }^{18,19}$ or twofold ${ }^{20}$ negatively charged in the bulk. Knowing from the STM experiments that the $\mathrm{V}_{\mathrm{Ga}}-\mathrm{Te}_{\mathrm{As}}$ complexes are the only compensating defects, and assuming that the complex formation does not change the charge balance, ${ }^{18}$ we can determine the charge state of $\mathrm{V}_{\mathrm{Ga}}$ in $n$-doped bulk GaAs. Our data are only consistent with a $-3 e$ charge of the Ga vacancy resulting in a $-2 e$ charge of the $\mathrm{V}_{\mathrm{Ga}}-\mathrm{Te}_{\mathrm{As}}$ complex.

The compensation in Te-doped GaAs is in many ways complementary to that in Si-doped GaAs. In Si-doped GaAs, the incorporation of $\mathrm{Si}_{\mathrm{As}}$ acceptors leads to the formation of Si pairs and ultimately Si clusters due to Coulomb interaction between the $\mathrm{Si}$ donors and acceptors. ${ }^{4}$ Tellurium is not incorporated as an acceptor; consequently, we do not observe the formation of neutral dopant pairs or clusters. In contrast, $\mathrm{V}_{\mathrm{Ga}}$ donor complexes are observed in Si-doped GaAs, too, with similar concentrations as in Te-doped $\mathrm{GaAs},{ }^{4}$ pointing to a universal formation mechanism independent of the $n$-type dopant element. The Ga vacancy formation energy is reduced with increasing $n$-type doping, the so-called Fermilevel effect, leading to an increased $\mathrm{V}_{\mathrm{Ga}}$ concentration independent of the dopant. ${ }^{18}$ Ultimately, the Coulomb interaction between negatively charged $\mathrm{Ga}$ vacancies and positively charged donors leads to the formation of donor Ga vacancy complexes.

In summary, we identified the microscopic origin of compensation in Te-doped GaAs with scanning tunneling microscopy. The $\mathrm{Te}_{\mathrm{As}}$ donors are exclusively compensated by the formation of Ga vacancy-donor complexes. This is explained with the Fermi level effect as the universal mechanism leading to vacancy formation and compensation of donors in $n$-type GaAs. A quantitative analysis of the defect concentration yields a $-3 e$ charge of the Ga vacancy in the bulk.

One author (J.G.) acknowledges a Feodor Lynen fellowship from the Alexander von Humboldt Foundation. This work was supported by the Director, Office of Science, Office of Basic Energy Sciences, Division of Materials Sciences and Engineering, of the U.S. Department of Energy, under contract No. DE-AC03-76SF00098.

${ }^{1}$ E. F. Schubert, Doping in III-V Semiconductors (Cambridge University Press, Cambridge, 1993).

${ }^{2}$ D. T. J. Hurle, J. Appl. Phys. 85, 6957 (1999).

${ }^{3}$ P. Ebert, Surf. Sci. Rep. 33, 121 (1999).

${ }^{4}$ C. Domke, P. Ebert, M. Heinrich, and K. Urban, Phys. Rev. B 54, 10288 (1996).

${ }^{5}$ K. Saarinen, P. Hautojärvi, A. Vehanen, R. Krause, and G. Dlubek, Phys. Rev. B 39, 5287 (1989)

${ }^{6}$ J. I. Landman, C. G. Morgan, J. T. Schick, P. Papoulias, and A. Kumar, Phys. Rev. B 55, 15581 (1997).

${ }^{7}$ W. Walukiewicz, Appl. Phys. Lett. 54, 2094 (1989).

${ }^{8}$ J. Gebauer, R. Krause-Rehberg, C. Domke, P. Ebert, and K. Urban, Phys. Rev. Lett. 78, 3334 (1997).

${ }^{9}$ P. Quadbeck, P. Ebert, K. Urban, J. Gebauer, and R. Krause-Rehberg, Appl. Phys. Lett. 76, 300 (2000).

${ }^{10}$ R. M. Feenstra, J. A. Stroscio, J. Tersoff, and A. P. Fein, Phys. Rev. Lett. 58, 1192 (1987).

${ }^{11}$ J. F. Zheng, X. Liu, N. Newman, E. R. Weber, D. F. Ogletree, and M. Salmeron, Phys. Rev. Lett. 72, 1490 (1994).

${ }^{12}$ C. Domke, P. Ebert, and K. Urban, Surf. Sci. 415, 285 (1998).

${ }^{13}$ G. Lengel, R. Wilkins, G. Brown, and M. Weimer, J. Vac. Sci. Technol. B 11, 1472 (1993).

${ }^{14}$ P. Ebert, M. Heinrich, M. Simon, C. Domke, K. Urban, C. K. Shih, M. B. Webb, and M. G. Lagally, Phys. Rev. B 53, 4580 (1996).

${ }^{15}$ C. Domke, P. Ebert, and K. Urban, Phys. Rev. B 57, 4482 (1998).

${ }^{16}$ K.-J. Chao, A. R. Smith, and C.-K. Shih, Phys. Rev. B 53, 6935 (1996).

${ }^{17}$ J. Gebauer, M. Lausmann, T. E. M. Staab, R. Krause-Rehberg, M. Hakala, and M. J. Puska, Phys. Rev. B 60, 1464 (1999).

${ }^{18}$ J. E. Northrup and S. B. Zhang, Phys. Rev. B 47, 6791 (1993); T. Y. Tan, H. M. You, and U. M. Gösele, Appl. Phys. A: Mater. Sci. Process. 56, 249 (1993).

${ }^{19}$ M. Luysberg, H. Sohn, A. Prasad, P. Specht, Z. Liliental-Weber, E. R. Weber, J. Gebauer, and R. Krause-Rehberg, J. Appl. Phys. 83, 561 (1998).

${ }^{20}$ H. Bracht, M. Norseng, E. E. Haller, K. Eberl, and M. Cardona, Solid State Commun. 112, 301 (1999). 